\title{
Analysis of Inorganic Nanoparticles by Single-particle Inductively Coupled Plasma Time-of-Flight Mass Spectrometry
}

\author{
Lyndsey Hendriks§, Alexander Gundlach-Graham, and Detlef Günther
}

§SCS-DSM Award for best poster presentation in Analytical Sciences

\begin{abstract}
Due to the rapid development of nanotechnologies, engineered nanomaterials (ENMs) and nanoparticles (ENPs) are becoming a part of everyday life: nanotechnologies are quickly migrating from laboratory benches to store shelves and industrial processes. As the use of ENPs continues to expand, their release into the environment is unavoidable; however, understanding the mechanisms and degree of ENP release is only possible through direct detection of these nanospecies in relevant matrices and at realistic concentrations. Key analytical requirements for quantitative detection of ENPs include high sensitivity to detect small particles at low total mass concentrations and the need to separate signals of ENPs from a background of dissolved elemental species and natural nanoparticles (NNPs). To this end, an emerging method called single-particle inductively coupled plasma mass spectrometry (sp-ICPMS) has demonstrated great potential for the characterization of inorganic nanoparticles (NPs) at environmentally relevant concentrations. Here, we comment on the capabilities of modern sp-ICPMS analysis with particular focus on the measurement possibilities offered by ICP-time-of-flight mass spectrometry (ICP-TOFMS). ICP-TOFMS delivers complete elemental mass spectra for individual NPs, which allows for high-throughput, untargeted quantitative analysis of dispersed NPs in natural matrices. Moreover, the multi-element detection capabilities of ICP-TOFMS enable new NP-analysis strategies, including online calibration via microdroplets for accurate NP mass quantification and matrix compensation.
\end{abstract}

Keywords: Matrix Effects · Nanoparticles · Single-Particle ICPMS · Size Distribution · Time-of-Flight Mass Spectrometry

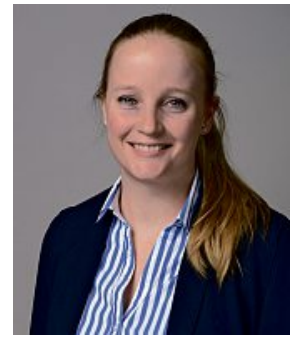

After growing up in the French speaking part of Switzerland, then moving to Zürich to study chemistry, Lyndsey Hendriks, whose mother tongue is English, is now almost perfectly trilingual. She obtained both her BSc and MSc degree in Chemistry at ETH Zürich in 2013, and 2015 respectively. Following a 6 months internship in Copenhagen focused on micro-and nanofabrication, she returned to Zürich to start her $\mathrm{PhD}$ in the analytical chemistry group of Prof. Dr. Detlef Günther. Her research focuses on nanoparticles detection and characterization using inductively coupled plasma mass spectrometry instruments.

${ }^{*}$ Correspondence: Dr. A. Gundlach-Graham Department of Chemistry and Applied Biosciences, ETH Zurich

Vladimir-Prelog-Weg 1, CH-8093 Zurich

E-mail: graham@inorg.chem.ethz.ch

\section{Introduction}

Single-particle inductively coupled plasma mass spectrometry (sp-ICPMS) was first reported in 2003 by Degueldre and Farvager for the analysis of dispersed natural colloids ${ }^{[1]}$ and later applied for analysis of micro-particle suspensions. ${ }^{[2,3]}$ Although the basics of the technique were established, it took several years for researchers to adopt the method because of limitations of existing quadrupole or sector-field ICPMS instruments. The trigger for renewed interest in sp-ICPMS was the rapid development and widespread use of engineered nanoparticles (ENPs), followed by the need for analytical techniques able to quantify and determine composition of individual NPs dispersed in natural matrices in order to investigate their impact on human health and ecosystems. ${ }^{[4-6]}$

\subsection{Potential Health Impact and Environmental Impact}

As the use of ENMs keeps expanding, their release into the environment is inevitable and will occur throughout several points in the nanomaterial 'life cycle', including during production, use, and disposal. For example, when socks and other pieces of sport clothing impregnated with silver NPs (AgNPs) are washed, the AgNPs used to kill bacteria and inhibit unpleasant odors are released and enter the waste water system, ${ }^{[7]}$ where they may or may not be adequately controlled by wastewater treatment plants. ${ }^{[8]}$ In another study, Gondikas et al. observed an increase in $\mathrm{TiO}_{2} \mathrm{NPs}$ in surface waters during the bathing season, as their use in sunscreen offers a direct route of release into the environment. [9]

In combination with presence of ENPs in surface waters, researchers have raised concerns that the very properties that make ENPs interesting - such as high surface reactivity for catalysis ${ }^{[10]}$ or ability to cross cell membranes for drug delivery - may also have a negative impact on human health and the environment. ${ }^{[11]}$ It is difficult to predict the future direction of nanosciences and the timescale over which particular developments will occur; however, the broad range of ENP applications indicates long-term use of nanotechnologies. ${ }^{[12,13]}$ In the short term, essential questions regarding safety and risk assessment of ENPs should be addressed. Past experiences with innovative products, such as chlorofluorocarbons (CFCs), dichlorodiphenyltrichloroethane (DDT), 
and methyl tert-butyl ether (MTBE), have revealed that the widespread use of such products is not always without consequences. While the public does not view NMs as a threatening technology, ${ }^{[14]}$ this position could change drastically when finding some negative effects on human health, which could hinder future application and development of nanotechnologies. ${ }^{[13,15]}$ Hence, it is appropriate to carry out risk and safety assessment studies, develop reliable and applicable analytical tools and procedures to measure NPs, and introduce appropriate safety regulations for workers, consumers, and the environment. To this end, several research commissions, such as the SNSF's 'Opportunities and Risks of Nanomaterials' national research program ${ }^{[16]}$ and the EU-funded the ACEnano (http:// www.acenano-project.eu/) aim to understand impacts of ENMs and develop analytical techniques and SOPs for NPrisk assessment.

\subsection{Analytical Techniques for Nano- material Characterization}

Along with the development of nanotechnologies, advanced analytical techniques and instrumentation have been (and continue to be) developed for the characterization of NPs and nanoscale features. Several overviews of these methods have been published. ${ }^{[5,17,18]}$ Synthetic NPs are typically characterized in terms of size, morphology, chemical composition, and surface activity, among other unique optical or electrical properties. Transmission or scanning electron microscopy (TEM/SEM) are the most popular and direct methods to determine physical dimensions and shape of ENPs, and can be combined with energy dispersive spectroscopy (EDS) or X-ray photoelectron spectroscopy (XPS) to provide compositions of individual ENPs. Atomic force microscopy (AFM) and scanning tunneling microscopy (STM) are used to examine topography and morphological features on structures. Apart from microscopy of individual particles, characterization of ensembles of ENPs is routinely done by dynamic light scattering (DLS) to provide size distribution information, or inductively coupled plasma mass spectrometry (ICPMS) or X-ray spectroscopies to measure elemental composition.

\subsection{Challenge with Environmental Samples}

While microscopy-based methods and bulk ensemble NP measurements are often adequate for synthetic NMcharacterization, these methods cannot be easily adapted to the characterization and quantification of ENPs dispersed in environmental matrices.
While electron microscopy techniques provide very high sensitivity (ability to detect ENPs ranging from 1 to $100 \mathrm{~nm}$ in diameter), these techniques are statistically weak and not well-suited for the detection of ENPs found at very low concentrations $\left(<\mu \mathrm{g} \mathrm{L}^{-1}\right)^{[6]}$ in the environment. ${ }^{[19]} \mathrm{In}$ fact, mass concentrations of ENPs are often lower than those of dissolved constituent elements, which limits the use of bulk element measurements without combination with a separation technique such as field-flow fractionation (FFF). Additionally, ENPs should be measured in complex matrices (such as surface waters, soils, or sewage sludge $\left.{ }^{[20]} . ..\right)$ that may contain natural nanoparticles (NNPs).[6] Native NNPs are formed by geochemical processes and are distributed throughout the natural soils, grounds, and surface waters, ${ }^{[21]}$ and may interfere with the analysis of ENPs. Natural nano-colloids or NPs with similar composition to their engineered analogues include metal oxide minerals, such as titanium dioxide, cerium oxide, silicon dioxide, and iron oxides. Despite similarities between natural and engineered NPs, specific properties such as chemical composition and size distribution may be used to differentiate between both types. ENPs often present a narrower size distribution than their naturally occurring counterparts, as they have been produced for specific, size-dependent properties. Finally, in 2011, the EU commission (2011/696/EU) adapted its choice of metrics for nanomaterials and now requires that analytical techniques provide particle number concentration (PNC) rather than a mass concentration. ${ }^{22]}$ Indeed, a low mass fraction of NPs may contain a large number of small nanoparticles, which are more reactive and penetrate organs and cells. ${ }^{[23]}$

Together, challenges of ENP analysis in environmental samples point toward a need for high-sensitivity, high-throughput, particle-counting methodologies that provide sufficient contrast to separate ENP analytes from complex backgrounds. Reliable risk assessment requires nanoanalysis methods that accurately measure size distributions, NP morphologies, chemical compositions, and PNCs. These analytical requirements remain a challenge and quite possibly will not be met by a single instrumental method; however, as discussed below, recent developments in sp-ICP-TOFMS are positioned to make substantial progress toward accurate detection of ENPs in complex matrices.

\section{4 sp-ICPMS, a Promising Technique for the Analysis of Inorganic Nanoparticles}

The high sensitivity, low detection limits, broad dynamic range, multi-isotope detection capabilities of ICPMS make it well-suited to tackle the special challenges of ENPs analysis in environmental samples. ${ }^{[17,24,25]}$ Because ICPMS can be used to detect metallic (e.g. Ag, Au,) and metal oxide (e.g. $\mathrm{Fe}_{3} \mathrm{O}_{4}, \mathrm{SiO}_{2}, \mathrm{TiO}_{2}, \mathrm{ZnO}, \mathrm{CeO}_{2}$ ) NPs, which are among the most widely used types of ENPs, interest in sp-ICPMS has grown rapidly in recent years. Evidence of the impact of sp-ICPMS can be seen in the volume of scientific publications as well as some review articles.[26,27]

\section{Theory of sp-ICPMS}

Single-particle (sp)-ICPMS enables the measurement of individual particles on a particle-to-particle basis and provides additional information that is otherwise unavailable from simple bulk analysis, such as PNC and particle mass distributions. Fig. 1 describes the basic principle behind sp-ICPMS measurements.

In sp-ICPMS, a dilute suspension of NPs is delivered to the ICP via standard pneumatic nebulization sample introduction. When an NP passes into the ICP source, it is efficiently vaporized, atomized, and ionized, and produces a temporally short burst of ions which passes through the mass analyzer. The typical duration of ion signal from an NP event is 200-500 $\mu \mathrm{s}$, and so, by collecting ICPMS signals at high time resolution, each NPgenerated signal is registered as an intensity spike on the ICPMS signal time trace. The frequency of these discrete NP-induced signals is proportional to particle number concentration (PNC), and the magnitude of these signal spikes correlates to the mass of each particle.

While the measurement strategy behind sp-ICPMS is straightforward, implementation to obtain accurate PNCs and NP sizes can be challenging. To accurately calibrate NP mass, the absolute detection efficiency (counts/ atom or counts/mass) for NPs of interest should be determined. While a number of approaches to measure this detection efficiency have been proposed, $[25,28]$ none are without shortcomings.[27,29] The most intuitive calibration approach is to measure the signal intensity of a series of NP standards of different sizes of the analyte of interest and establish a calibration curve. Then - the same way one would do with solution-based ICPMS analysis - one could measure unknown NP samples and calibrate size/mass of the particles. However, even though this approach sounds the most straightforward, it is limited by the availability of wellcharacterized monodisperse NP standards and solution-based element standards are typically used for sensitivity calibration. 


\section{a) Conventional liquid ICPMS}
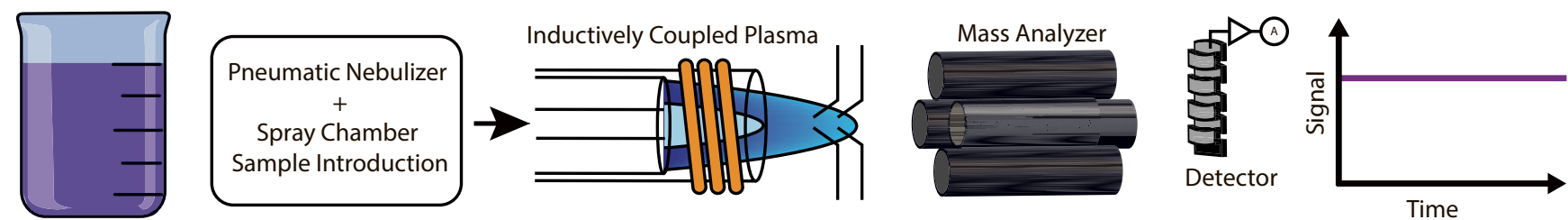

\section{b) Single-particle ICPMS}

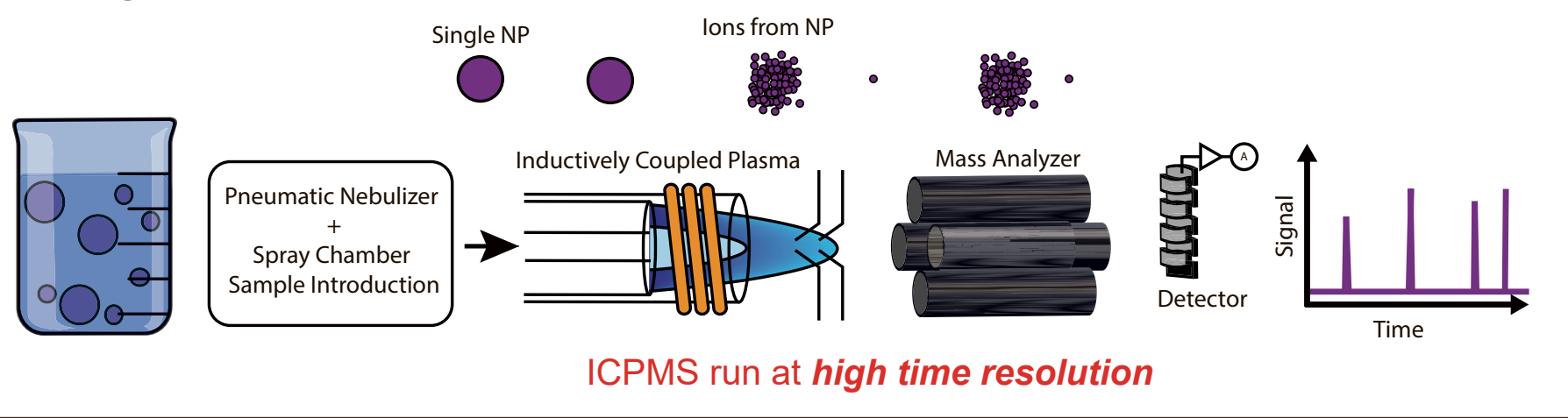

Fig. 1. a) Conventional solution-based ICPMS: The liquid sample is introduced into the ICPMS by using a nebulization system, which generates an aerosol of polydisperse droplets. The droplets are transported into the ICP by an argon gas flow. In the plasma, the droplets are evaporated, and the analyte they contain then undergoes atomization and ionization. In the final step, the ions are extracted into the mass spectrometer, where a useful analytical signal is recorded. Assuming that the solution is homogenous, a steady signal will be observed. b) sp-ICPMS: A dilute suspension of NPs is introduced into the plasma via the same nebulization system as above. Each NP is digested in the ICP and generates a group of analyte ions, that are spatially and temporally dense. If the ICPMS is run at high time resolution, the signal from each individual NP will be measured as a signal spike above the baseline. When using quadrupole or sector-field ICPMS, only one isotope per measurement can be detected and this is a significant drawback.

In this work, we make use of a microdroplet-based calibration strategy ${ }^{[30,31]}$ to determine NP mass. In this approach, microdroplets containing known concentrations of standard salt solutions are introduced into the ICP as dried aerosol particles following desolvation of droplets in helium drying gas. Calibrant droplets can be thought of as a NP proxy, ${ }^{[32]}$ and, because droplet size is measured, the mass of analyte in each dried droplet is known. Through timeresolved sp-ICP-TOFMS analysis, singledroplet signals are recorded and absolute detection efficiencies for all elements in the droplets are determined. Because the ICP is a robust and high dynamic range atomization and ionization source, analyte responses are linear across several orders of magnitude of analyte mass and singlepoint calibration is adequate.

\section{Results and Discussion: sp-ICP- TOFMS}

\subsection{Size Determination and Linear Dynamic Range}

Gold NPs can be used for cancer therapy as they present low cell toxicity and present useful optical properties. ${ }^{[33]}$ In this case, as they are commercially available, monodisperse and well-characterized, they were used for size determination by sp-ICPMS with microdroplets. In Fig. 2, monodisperse 30-nm gold ENPs were measured by TEM (a) and then characterized using sp-ICP-TOFMS and microdroplets for particle mass calibration, resulting in a size distribution centered at $30 \mathrm{~nm}$ (b). In addition, we demonstrate the linear dynamic range from $30 \mathrm{~nm}$ to 250 $\mathrm{nm}$ (see Fig. 2c), with a limit of detection in terms of diameter of $19 \mathrm{~nm}$ (i.e. $22 \mathrm{ag}$ ). In an earlier study, Degueldre et al. obtained a linear response for Au NPs ranging from 80 to $200 \mathrm{~nm} ;{ }^{[3]}$ higher sensitivity and high-dynamic range detection electronics of the ICP-TOFMS instrument enable the dynamic range improvement reported here.

\subsection{Multi-element Advantage of sp- ICP-TOFMS: Analysis of a Mixture of Au NPs, Ag NPs and Ag/Au Core- shell NPs}

A drawback of sp-ICPMS with conventional ICPMS instruments that employ quadrupole or sector-filed mass analyzers is that only signals from one isotope can be measured per particle. This is particularly disadvantageous when analyzing NPs with multi-element composition, such as core-shell NPs, or when trying to differentiate ENPs from NNPs based on multi-element composition. To detect multi-element NPs by sp-ICPMS, a simultaneous mass analyzer, such as TOFMS, must be used in combination with the ICP ion source. In results provided here, we use a newly introduced ICP-TOFMS mass spectrometer (icpTOF, TOFWERK AG, Switzerland), ${ }^{[34,35]}$ which enables quasi-simultaneous detection over the whole elemental mass range at time resolutions down to $30 \mu \mathrm{s}$. These attractive properties can be particularly useful for screening of ENPs in natural matrices, as well as the analysis of samples without a priori knowledge of the NPs or their

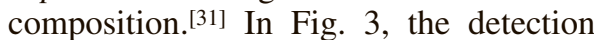
capabilities of the ICP-TOFMS instrument are illustrated by monitoring the timetraces of silver and gold isotopes, which enables the detection of pure silver and gold NPs, as well as silver-shelled goldcore NPs. Using conventional quadrupole or sector-field instruments, in which only one isotope is measured per nanoparticle, the above quantitative analysis would not be possible. Sequential mass analysis is a significant disadvantage for measurement of short transients like from multicomponent NPs. On the other hand, spICP-TOFMS is a promising technique for high-throughput and untargeted analysis of diverse NPs.

In addition to measuring multiple NP types simultaneously, multi-elemental data provided by ICP-TOFMS may be 


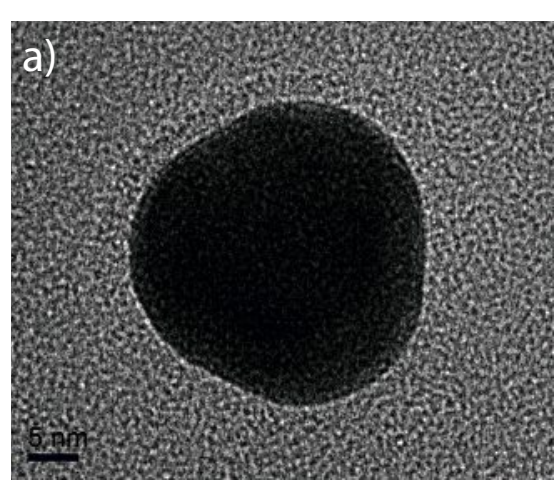

b)
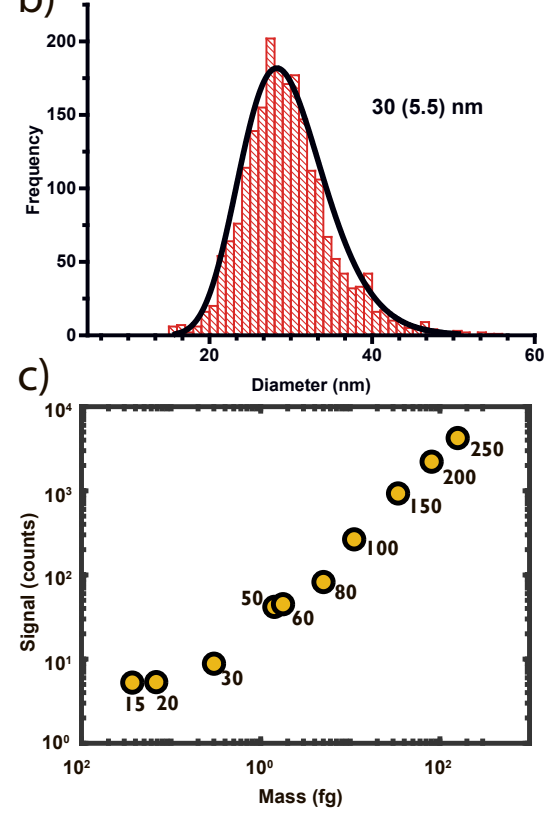

Fig. 2. a) TEM image of a 30-nm diameter gold NP. b) Size distribution of the $30 \mathrm{~nm}$ gold NPs, measured by sp-ICP-TOFMS in microdroplets and calibrated with gold standards in microdroplets. c) Linear response for gold NPs ranging from $30 \mathrm{~nm}$ to $250 \mathrm{~nm}$ diameter with a limit of detection of $19 \mathrm{~nm}(22 \mathrm{ag})$.

used to differentiate between natural and engineered nanoparticles because total chemical composition of the particle types may predictably differ. For example, in a research collaboration between researchers from the University of Vienna and our lab, we demonstrated that $\mathrm{CeO}_{2}$ ENPs can be quantitatively distinguished from naturally occurring Ce-containing colloids based on conserved multi-element fingerprints of the NPs measured by sp-ICP-TOFMS. [36] Importantly, measurement of ${ }^{140} \mathrm{Ce}$ only, would not allow for Ce-containing NNPs to be differentiated from the $\mathrm{CeO}_{2}$. Singleparticle-ICP-TOFMS uniquely enables the distinction of NPs from dissolved ions and natural particle background species in a complex environmental matrix.

\subsection{Application of sp-ICP-TOFMS for Food Sample Analysis}

While sizing of ENPs in aqueous samples is relatively straightforward by

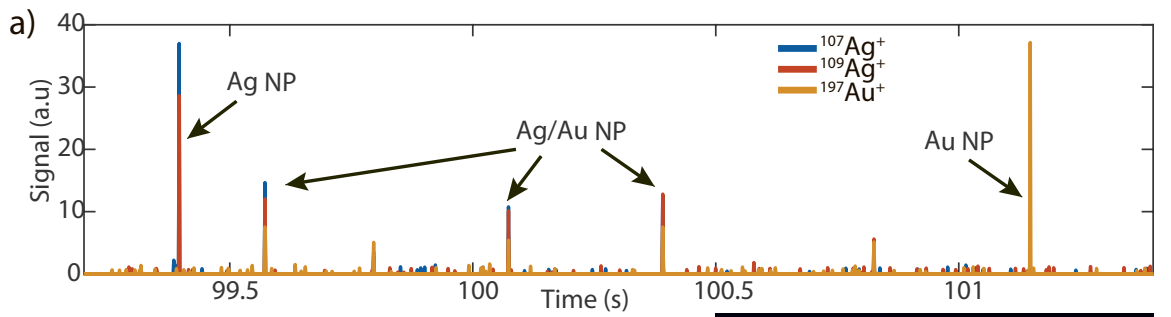

b) $60 \mathrm{~nm}$ Ag shelled Au spheres
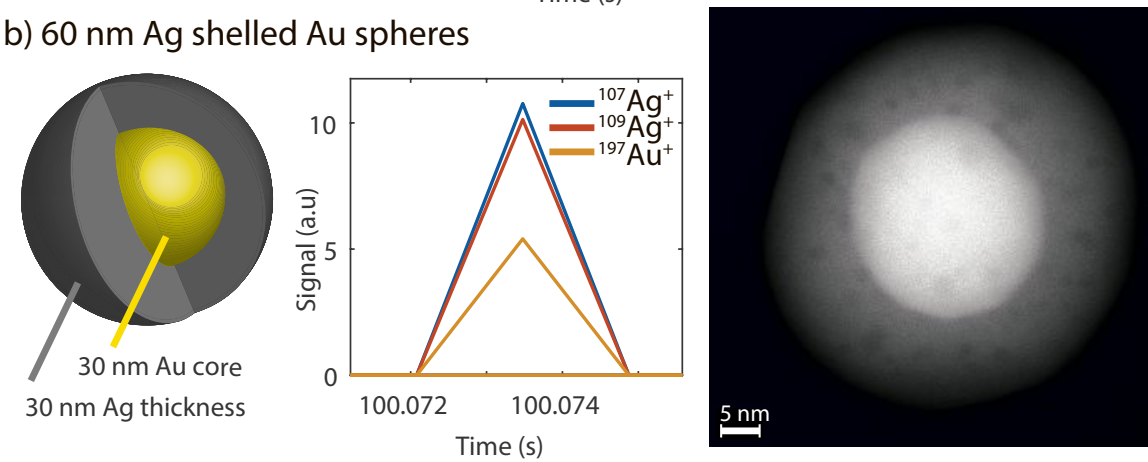

Fig. 3. a) sp-ICP-TOFMS time trace of a mixture of 60-nm Ag NPs, 50-nm Au NPs and Ag/Au coreshell NPs. With ICP-TOFMS, all NP types can be measured and distinguished in a single run. b) Zoom-in of the time-trace and TEM picture of a silver-shelled gold-core nanosphere.
sp-ICPMS methods (see Fig. 2), accurate mass determination of NPs in natural systems is more challenging. In these complex matrices, low PNCs, NP-size polydispersity, NP aggregation, and influence of the matrix on analytical sensitivity all complicate sp-ICPMS measurements. It has been shown that mismatch between solvents used for elemental standards and matrix composition of NP suspensions can cause NP-mass determination errors of up to $90 \% .{ }^{[27]}$ This is particularly a problem for real-world samples in which matrices cannot be removed and conventional matrix-matched calibration is impractical or sp-ICP-TOFMS can impact accuracy and robustness of sp-ICPMS analysis.

In recent work, we have combined introduction first reported by Ramkorunimpossible. Here, again, developments in ICP-TOFMS with a dual-sample
Schmidt et al. ${ }^{[37]}$ In this system (see Fig. 4), microdroplets with known element amounts are introduced concurrently with the NP samples, which are introduced via a conventional nebulizer and spray chamber system. Because dried-droplet calibrant aerosols are introduced along with the matrix-containing NP suspension, both dried droplets and analyte NPs experience the same plasma conditions. This type of online microdroplet 'standard addition' tactic can be used to compensate for plasma-related matrix effects. Here again, the quasi-simultaneous multi-isotope detection with TOFMS is critical because microdroplet signals are identified and differentiated from NP signals based on multi-elemental composition of the droplets. This approach combines advantages of sample-introduction with a conventional pneumatic nebulizer,

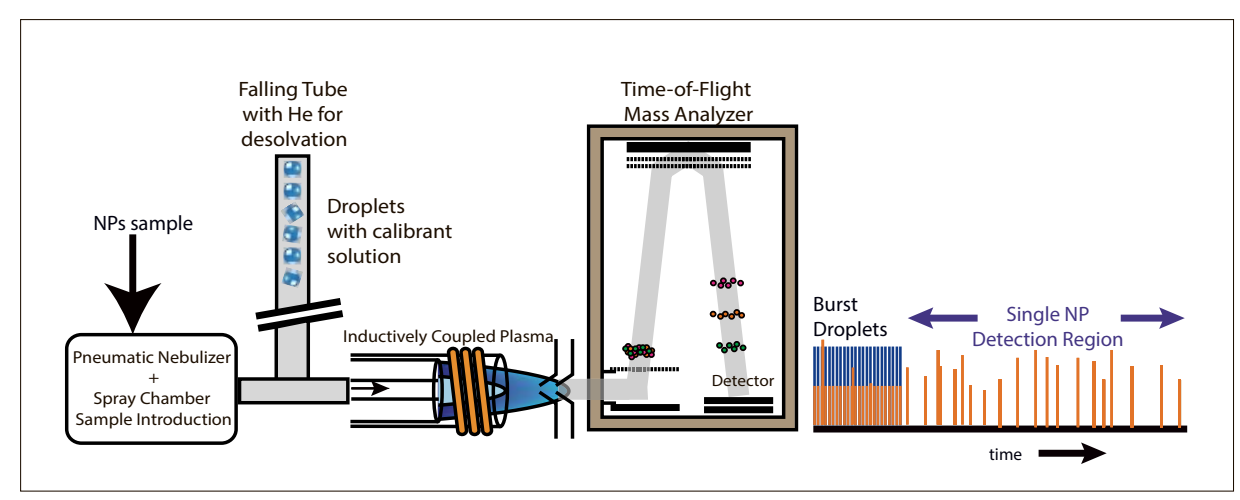

Fig. 4. Schematic of the dual sample introduction approach combined with sp-ICP-TOFMS to characterize NPs in complex matrix. The microdroplets are 'added' to the signal of the aspirated NP-containing sample, thereby the dried aerosol from the microdroplets and NPs experience the same plasma conditions. The signals from the droplets can be differentiated from NP signals in the time trace by monitoring non-analyte isotopes. 
including large volume introduction and rapid switching between different NP-containing samples, with the use of microdroplets for calibration, which allows for the analysis of a wide range of NPs.

To demonstrate the utility of the dual sample-introduction setup, we investigated the sizing of gold NPs spiked into beverages, namely orange juice and milk. Food matrices were investigated because exposure to nanomaterials through food products is increasing. ${ }^{[38]}$ In this study, we spiked nominally $150-\mathrm{nm}$ Au NPs into diluted orange juice and milk, and used a 200-ppb multi-elemental solution in the microdroplets for mass-sensitivity calibration. As shown in Fig. 5, when using 1:10 diluted milk as a matrix, signal intensities were decreased by $\sim 50 \%$ for both the calibrant droplets and NP signals. Because the calibrant and analyte NP were affected in a similar manner, the signal attenuation due to the milk matrix could be compensated to a large extent and Au NPs were sized more accurately than they would have been without the microdroplet standard addition approach. For the orange juice matrix, minimal signal enhancement was observed and Au NPs sizing was identical to that obtained in a water matrix.

Demonstration of matrix-effect compensation by this online microdrop let standard addition approach indicates that sp-ICP-TOFMS could be used to quantitatively measure ENPs in a suite of different matrices without need for adjustment of calibration strategy. Moreover, this approach should be extendable to mass determination of a range of NP types in a single run, thus greatly improving measurement throughput. While further investigation is still required, results here indicate that sp-ICP-TOFMS could be an enabling technology for comprehensive inorganic NP measurements in important high-matrix, low-volume samples such as biological fluids.

\section{Conclusion}

In this overview of sp-ICPMS, we have discussed its potential for the analysis of NPs in environmental samples, including detection, characterization and quantification. While single-particle ICPMS is now a well-accepted technique for analysis of dispersed NPs, the use of sequential mass analyzers for singleparticle analysis has many limitations, especially in the ability to distinguish between ENPs and a high background of NNPs, colloids, and dissolved elements. On the other hand, sp-ICP-TOFMS analysis allows for untargeted NP detection because it provides complete elemental mass spectra for individual
NPs, which has proven to be critical when dealing with mixtures of ENPs and NNPs. Additionally, sp-ICP-TOFMS is ideally suited to monitor transformation processes of NPs, which can significantly impact NP toxicity. The multi-elemental capabilities of TOFMS also enable new creative setups for single particle analysis as demonstrated with the dual inlet calibration approach, which proved to be a successful strategy to manage matrix effects that can otherwise lead to inaccurate NP sizing. Despite the many advantages of ICPTOFMS for NP detection, a remaining challenge for instrument development is improved sensitivity. When compared to other ICPMS instruments, such as sectorfield ICPMS instruments, ICP-TOFMS provides between 10 and 100 times less sensitivity for single-isotope detection. ${ }^{[35]}$ Continued efforts to improve sensitivity and data processing, followed by more application-directed studies using sp-ICPTOFMS, will help researchers to gain a better understanding of the measurement possibilities offered by Time-of-Flight ICPMS, and should establish sp-ICPTOFMS as a powerful analytical technique for the measurement of ENPs in real-world samples.

\section{Acknowledgments}

This work was financially supported by the Swiss National Science Foundation (SNSF), Project no. 200021 162870/1. A. GundlachGraham acknowledges financial support through the Ambizione grant of the SNSF; Project number PZ00P2_174061. The authors thank Roland Mäder from the ETH mechanical workshop for manufacturing custom pieces necessary for the microdroplet introduction system and the dual inlet setup. The authors also thank Dr. Frank Krumeich for SEM/TEM imaging.

Received: February 2, 2018

[1] C. Degueldre, P. Y. Favarger, Colloids Surf., A 2003, $217,137$.

[2] C. Degueldre, P. Y. Favarger, C. Bitea, Anal. Chim. Acta 2004, 518, 137.

[3] C. Degueldre, P. Y. Favarger, S. Wold, Anal. Chim. Acta 2006, 555, 263.

[4] A. Scheffer, C. Engelhard, M. Sperling, W. Buscher, Anal. Bioanal. Chem. 2008, 390, 249; M. Hassellöv, R. Kaegi, in 'Environmental and Human Health Impacts of Nanotechnology', John Wiley \& Sons, Ltd, 2009, p. 211, DOI: 10.1002/9781444307504.ch6; B. Nowack, T. D. Bucheli, Environ. Pollut. 2007, 150, 5; M. N. Moore, Environ. Int. 2006, 32, 967.

[5] M. Hassellov, J. W. Readman, J. F. Ranville, K. Tiede, Ecotoxicology 2008, 17, 344; A. LopezSerrano, R. M. Olivas, J. S. Landaluze, C. Camara, Anal. Methods 2014, 6, 38.

[6] M. D. Montano, G. V. Lowry, F. von der Kammer, J. Blue, J. F. Ranville, Environ. Chem. 2014, 11, 351.

[7] J. R. Morones, J. L. Elechiguerra, A. Camacho, K. Holt, J. B. Kouri, J. T. Ramirez, M. J.

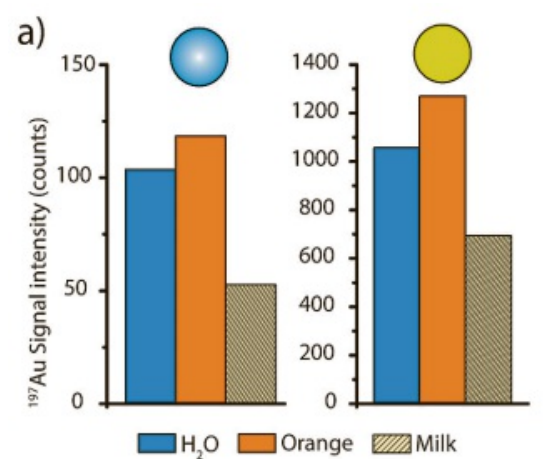

b) $\square$ Size distribution of Au NPs in $\mathrm{H}_{2} \mathrm{O}$

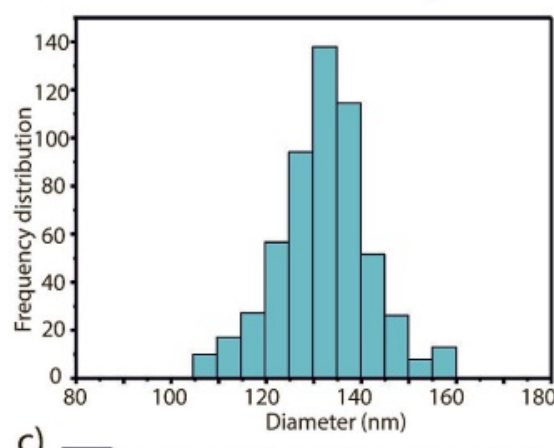

c)

Size calibration using sensitivity determined in $\mathrm{H}_{2} \mathrm{O}$ Size calibration using sensitivity determined in corresponding matrix
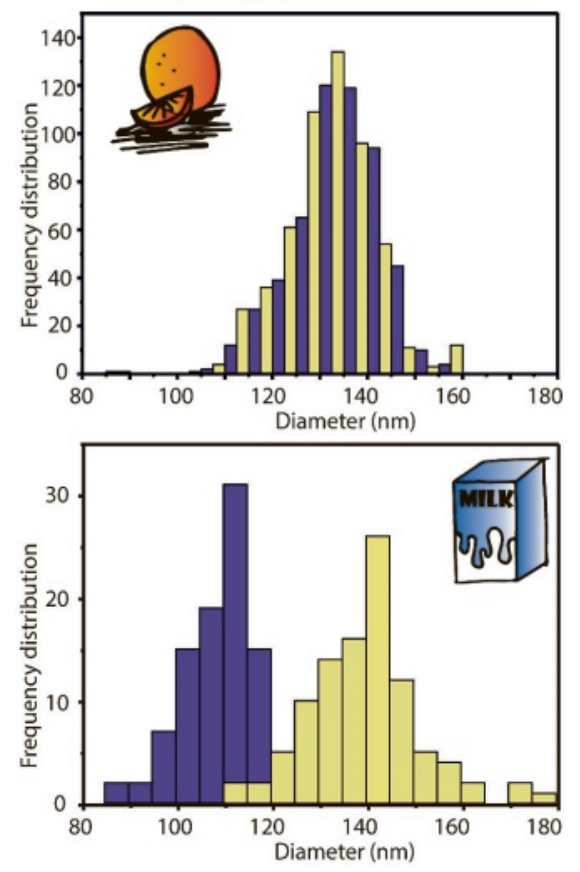

Fig. 5. a) Average gold signal intensities from microdroplets (left) and average Au NP signal intensities (right) with different matrices were aspirated through the pneumatic nebulizer. b) Size calibration of Au NPs in water. c) Size calibrations of same Au NPs in orange juice and milk matrices. If gold sensitivities are determined from droplets introduced with sample matrices, NP size can be determined accurately regardless of matrix effects. In contrast, if sensitivities are determined without sample present (i.e. with water aspirated), inaccurate sizing may result - as in the case of the milk matrix. 
Yacaman, Nanotechnology 2005, 16, 2346; D M. Mitrano, E. Rimmele, A. Wichser, R. Erni, M. Height, B. Nowack, ACS Nano 2014, 8, 7208.

[8] L. X. Y. Li, M. Stoiber, A. Wimmer, Z. L. Xu, C. Lindenblatt, B. Helmreich, M. Schuster, Environ. Sci. Technol. 2016, 50, 6327; R. Kaegi, A. Voegelin, C. Ort, B. Sinnet, B. Thalmann, J. Krismer, H. Hagendorfer, M. Elumelu, E. Mueller, Water Res. 2013, 47, 3866.

[9] A. Gondikas, F. von der Kammer, R. Kaegi, O. Borovinskaya, E. Neubauer, J. Navratilova, A. Praetorius, G. Cornelis, T. Hofmann, Environ. Sci.: Nano 2018, 5, 313.

[10] M. Haruta, 3rd World Congress on Oxidation Catalysis 1997, 110, 123; G. J. Hutchings, Gold Bulletin 2004, 37, 3 .

[11] A. Nel, T. Xia, L. Madler, N. Li, Science 2006, 311, 622; K. Savolainen, H. Alenius, H. Norppa, L. Pylkkänen, T. Tuomi, G. Kasper, Toxicology 2010, 269, 92; F. Gottschalk, B. Nowack, J. Environ. Monit. 2011, 13, 1145.

[12] N. Staggers, T. McCasky, N. Brazelton, R. Kennedy, Nursing Outlook 2008, 56, 268.

[13] D. Parr, Trends in Biotechnology 2005, 23, 395.

[14] Nanopinion, Monitoring public opinion on Nanotechnology in Europe, http://nanopinion. archiv.zsi.at/, accessed 24.01.2018.

[15] N. M. d. S. Cameron, in 'Progress in Convergence: Technologies for Human Wellbeing', Vol. 1093, John Wiley \& Sons, 2006, p. 280, DOI: 10.1196/annals.1382.019.

[16] National Presearch Programme NRP 64: Oppportunities and Risks of Nanomaterials, http://www.nrp64.ch/en, accessed 24.01.2018.

[17] P. Krystek, A. Ulrich, C. C. Garcia, S. Manohar, R. Ritsema, J. Anal. At. Spectrom. 2011, 26 , 1701.

[18] F. Laborda, E. Bolea, G. Cepria, M. T. Gomez, M. S. Jimenez, J. Perez-Arantegui, J. R. Castillo, Anal. Chim. Acta 2016, 904, 10.

[19] M. F. Hochella, D. Aruguete, B. Kim, A. S Madden, in 'Nature's Nanostructures', Eds. A. S. Barnard, H. Guo, Chap. 1, 2012.
[20] B. Kim, M. Murayama, B. P. Colman, M. F. Hochella, J. Environ. Monit. 2012, 14, 1129.

[21] M. F. Hochella, S. K. Lower, P. A. Maurice, R. L. Penn, N. Sahai, D. L. Sparks, B. S. Twining, Science 2008, 319, 1631; R. F. Domingos, M. A. Baalousha, Y. Ju-Nam, M. M. Reid, N. Tufenkji, J. R. Lead, G. G. Leppard, K. J. Wilkinson, Environ. Sci. Technol. 2009, 43, 7277; F. von der Kammer, P. L. Ferguson, P. A. Holden, A. Masion, K. R. Rogers, S. J. Klaine, A. A. Koelmans, N. Horne, J. M. Unrine, Environ. Toxicol. Chem. 2012, 31, 32; M. F. Hochella, M. G. Spencer, K. L. Jones, Environ. Sci.: Nano 2015, 2, 114.

22] Communication from the Commission to the European Parliament, the Council and the European Economic and Social Committee on the Second Regulatory Review on Nanomaterials, https://ec.europa.eu/health// sites/health/files/nanotechnology/docs/ swd_2012_288_en.pdf.

[23] L. Braydich-Stolle, S. Hussain, J. J. Schlager, M. C. Hofmann, Toxicol. Sci. 2005, 88, 412.

[24] M. S. Jiménez, M. T. Gómez, E. Bolea, F. Laborda, J. Castillo, Int. J. Mass Spectrom. 2011, 307, 99; J. Tuoriniemi, G. Cornelis, M. Hassellov, Anal. Chem. 2012, 84, 3965.

[25] H. E. Pace, N. J. Rogers, C. Jarolimek, V. A. Coleman, E. P. Gray, C. P. Higgins, J. F. Ranville, Environ. Sci. Technol. 2012, 46, 12272.

[26] F. Laborda, E. Bolea, J. Jimenez-Lamana, Anal. Chem. 2014, 86, 2270; F. Laborda, E. Bolea, J. Jiménez-Lamana, Trends Environ. Anal. Chem. 2016, 9, 15; 'Themed Collection: 5 years of nanomaterial analysis by means of ICP-MS', Eds. B. Meermann, F. Laborda, J. Anal. At. Spectrom. 2015.

[27] M. D. Montano, J. W. Olesik, A. G. Barber, K. Challis, J. F. Ranville, Anal. Bioanal. Chem. 2016, 408, 5053.

[28] H. E. Pace, N. J. Rogers, C. Jarolimek, V. A. Coleman, C. P. Higgins, J. F. Ranville, Anal. Chem. 2011, 83, 9361; J. W. Olesik, P. J. Gray, J. Anal. At. Spectrom. 2012, 27, 1143.
[29] S. Gschwind, M. Aja Montes, D. Günther, Anal. Bioanal. Chem. 2015, 407, 4035.

[30] C. C. Garcia, A. Murtazin, S. Groh, V. Horvatic, K. Niemax, J. Anal. At. Spectrom. 2010, 25, 645; S. Gschwind, H. Hagendorfer, D. A. Frick, D. Günther, Anal. Chem. 2013, 85, 5875.

[31] O. Borovinskaya, S. Gschwind, B. Hattendorf, M. Tanner, D. Günther, Anal. Chem. 2014, 86, 8142 .

[32] J. W. Olesik, Appl. Spectrosc. 1997, 51, 158A O. Borovinskaya, M. Aghaei, L. Flamigni, B. Hattendorf, M. Tanner, A. Bogaerts, D. Günther, J. Anal. At. Spectrom. 2014, 29, 262.

[33] S. Jain, D. G. Hirst, J. M. O'Sullivan, Br. J. Radiol. 2012, 85, 101; Z. Z. J. Lim, J. E. J. Li, C. T. Ng, L. Y. L. Yung, B. H. Bay, Acta Pharmacol. Sin. 2011, 32, 983.

[34] O. Borovinskaya, B. Hattendorf, M. Tanner, S. Gschwind, D. Günther, J. Anal. At. Spectrom. 2013, 28, 226.

[35] L. Hendriks, A. Gundlach-Graham, B. Hattendorf, D. Günther, J. Anal. At. Spectrom. 2017, 32, 548 .

[36] A. Praetorius, A. Gundlach-Graham, E. Goldberg, W. Fabienke, J. Navratilova, A. Gondikas, R. Kaegi, D. Günther, T. Hofmann, F. von der Kammer, Environ. Sci.: Nano 2017, 4, 307.

[37] B. Ramkorun-Schmidt, S. A. Pergantis, D. Esteban-Fernandez, N. Jakubowski, D. Günther, Anal. Chem. 2015, 87, 8687.

[38] K. Ramos, M. M. Gomez-Gomez, C. Camara, L. Ramos, Talanta 2016, 151, 83; T. P. J. Linsinger, Q. Chaudhry, V. Dehalu, P. Delahaut, A. Dudkiewicz, R. Grombe, F. von der Kammer, E. H. Larsen, S. Legros, K. Loeschner, R. Peters, R. Ramsch, G. Roebben, K. Tiede, S. Weigel, Food Chem. 2013, 138, 1959; M. Mattarozzi, M. Suman, C. Cascio, D. Calestani, S. Weigel, A. Undas, R. Peters, Anal. Bioanal. Chem. 2017, 409, 63 . 